\section{Lettera aperta ai componenti di un team che non ce la fa a decollare: quello per gli accessi vascolari (A-V)}

Il team per gli accessi vascolari, possibilmente multidisciplinare, è una realtà la cui presenza sul nostro territorio nazionale non è neppur definibile a macchia di leopardo: magari! Eppure tutti abbiamo ormai passato decenni a dibattere che il nefrologo deve essere l'operatore sanitario "regista" di questo team e, quindi, colui che ne organizza l'esistenza. Viceversa sono ormai svariati anni che il team per gli A-V per emodialisi sembra aver lo stesso destino di quello di una "ragazza dalle belle ciglia: tutti la vogliono ma nessuno la piglia" e l'A-V per emodialisi continua a essere "il tallone d'Achille dell'emodialisi".

La frequenza di alcuni convegni sull'argomento (il Corso Educazionale della SIN, un Advanced Workshop tenutosi il 28 Ottobre in quel di Pistoia, il Congresso Nazionale del GdS degli A-V a Lucca, ecc.) mi ha ridato la voglia e la forza di esplicitare e chiedere a chi ha più know-how di me in merito ad alcuni punti che seguono. Possibile non si riesca a venire a capo?

1) All'interno di quel che esiste e possa comunque essere definito come team per gli $\mathrm{A}-\mathrm{V}$, il design dell'accesso vascolare dovrebbe essere sempre fatto in presenza di un nefrologo, ovvero di colui che assieme agli infermieri di dialisi avrà la facoltà/responsabilità di usare e mantenere questo prezioso bene del paziente. Mi sembra pertanto ovvio che il nefrologo dovrebbe essere sempre presente assieme al chirurgo vascolare, piuttosto che all'ecografista/radiologo, nella fase in cui viene eseguito il mapping pre-FAV. In tal modo a quello che potrebbe essere definito come tavolo di contrattazione per la creazione di un A-V non deve/può mancare l'esperienza essenziale della sala dialisi. Questo per cercare di ottenere -almeno sulla carta- il migliore progetto dell'A-V nell'ottica dell'uso che ne dovrà esser fatto in sala dialisi.

2) La manutenzione della FAV dovrebbe essere il $1^{\circ}$ comandamento per la durata degli accessi vascolari. Qui le figure pivotali si invertono ma è come in una moltiplicazione, vale la proprietà commutativa, ovvero cambiando l'ordine dei fattori il prodotto non cambia né cambia il risultato. L'infermiere di dialisi è senza dubbio, seguito a ruota dal nefrologo in dialisi, la figura che ha la maggiore responsabilità (onori e oneri) nella durata dell'accesso. Pertanto dovrebbero essere creati dei corsi obbligatori (dalla SIN) per queste figure (nefrologo e infermiere in dialisi); pensando all'attuale rebound della formazione permanente degli operatori sanitari non posso non chiedermi se gli ECM maturati da infermieri e nefrologi in dialisi non dovrebbero prevedere un'elevata percentuale di crediti ottenuti a corsi che trattino questo argomento (nota polemica: sicuramente più di quelli ottenuti da corsi su anti-incendio e anti-corruzione o per la gestione/ sistemazione dei farmaci negli armadi, ecc.).

3) Non è pensabile oggigiorno che in un team per gli A-V possa mancare un radiologo interventista e, laddove manchi un nefrologo capace di creare $\mathrm{A}-\mathrm{V}$, un chirurgo vascolare. Non basta. Queste figure, chirurgo vascolare e radiologo interventista, non possono non avere un'infarinatura di ciò che serve in sala dialisi: ancora corsi ad hoc e ancora SIN per promuovere tra tutte queste figure un'osmosi tale da permettere una crescita contemporanea con maggiore, ma che dico MIGLIORE, collaborazione. Un esempio. In un'epoca di High Technology Assessment e di angioplastiche percutanee (PTA), com'è possibile che quando mandiamo un paziente a correggere/trattare una stenosi dell'A-V con una PTA il risultato sia espresso ancora mediante parole e aggettivi? Perché, viceversa, non mediante anche numeri (sicuramente più confrontabili!). I numeri, per esempio, potrebbero essere quelli espressi dalle misurazioni delle pressioni trans-stenotiche "prima" della PTA e "dopo". Ciò, oltre a darci modo di oggettivare il risultato delle importanti manovre di chi è addetto ad eseguire la PTA del vaso stenotico, potrebbe dare, a lungo andare, anche uno strumento in grado di controllare l'operato, ovviamente in senso buono e costruttivo, ma soprattutto utile nel monitorare/interpretare meglio nel tempo il recoil o la recidivanza di tali stenosi.

4) Si stanno muovendo i primi passi (anche nella mia Regione) affinché le manovre di mapping nella creazione o nella correzione vascolare siano sempre meno pericolose per i pazienti e per i loro reni (penso ai pazienti in pre-dialisi in odore di una prima FAV). In questo un'angiografia alla $\mathrm{CO} 2$ invece che al m.d.c. potrebbe/potrà essere una valida alternativa? Siamo molto lontani da questa realtà? Quali i principali ostacoli a ciò?

Visti questi miei interrogativi, c'è qualcuno che, tastiera alla mano, se la sente di spiegarmi meglio e/o fare un punto della situazione? Ma, soprattutto, c'è qualcuno in grado di indicare/pronosticare quale sia la via più breve per raggiungere tutto quanto di meglio per gli A-V dei nostri pazienti in emodialisi?

\section{Disclosures}

Financial support: No financial support was received for this submission.

Conflict of interest: The author has no conflict of interest.

Marco Lombardi

Editor in Chief

Giornale di Tecniche Nefrologiche e Dialitiche

Published online: February 2, 2017 